\title{
Driver Fatigue Estimation Using Image Processing Technique
}

\author{
Vijayalaxmi \\ Vignan Institute of Technology \& Science, Vignan Hills, Nalgonda District, Hyderabad, Telangana, India \\ E-mail: laxmi81181@gmail.com \\ D. Elizabeth Rani \\ Gitam Institute of Technology, Gitam University, Vishakapatnam, Andhra Pradesh, India
}

\begin{abstract}
A Fatigue Detection system has been developed using non-intrusive vision based approach. The system uses a Logitech USB camera which points towards driver's face and monitors face and eyes to detect driver fatigue. The system is developed on Linux operating system and used DM3730 processor as hardware. The algorithm is developed to estimate whether eyes are open or closed and fatigue is estimated using PERCLOS method. Normal human blinks eyes 12 times in a minute. If the eyes are closed for 15 consecutive frames in a minute or if PERCLOS $>80 \%$ than system issues warning to stop the vehicle. The algorithm is tested on 45 different persons i.e., 15 women, 15 men and 15 persons wearing spectacles and the detection rate is $99.2 \%$. The system takes $<5 \mathrm{~ms}$ of time to detect whether eyes are open or closed and the hardware used is small in size and easily implementable.
\end{abstract}

Index Terms-DM3730, Eye, Face, Fatigue, Haar, PERCLOS, VITS.

\section{INTRODUCTION}

The need of public transportation is growing at a rapid rate which has increased road accident statistics. There are number of safety devices used in vehicles to protect the driver like seat belts, air bags, speed brake, etc. These devices always act after the accident has happened. There is lot of research happened to estimate driver fatigue and alert the driver to prevent accidents on road. The driver fatigue detection systems are broadly classified into two types Intrusive and Non-intrusive system. The block diagram of driver fatigue detection system is shown in Fig 1 .

W. W. Wierwille, S. S. Wreggit, et.al,[1] proposed monitoring changes in physiological characteristics like ECG,EEG, skin temperature, head movement to estimate driver fatigue. The drawback of this approach is, it causes distraction, non-realistic and disturbance to the driver.

Artaud et.al,[2] and Mabbott et.al,[2] proposed a method of sensing driver response by placing sensors on steering wheel and back of the seat. The drawback of this approach, it fails if driver wears gloves and performance of sensors placed on back seat reduces with time.
Boyraz.P.,Leicester,Hansen J.H.L [3], proposed a method of sensing vehicle response to measure uncertainty in steering wheel. The drawback of this approach is, it is limited to vehicle type and driver condition. Mabbottt et.al[2], proposed a method were driver response is monitored requesting the driver to send feedback continuously. The drawback of this approach is driver gets tiresome and feels annoying.

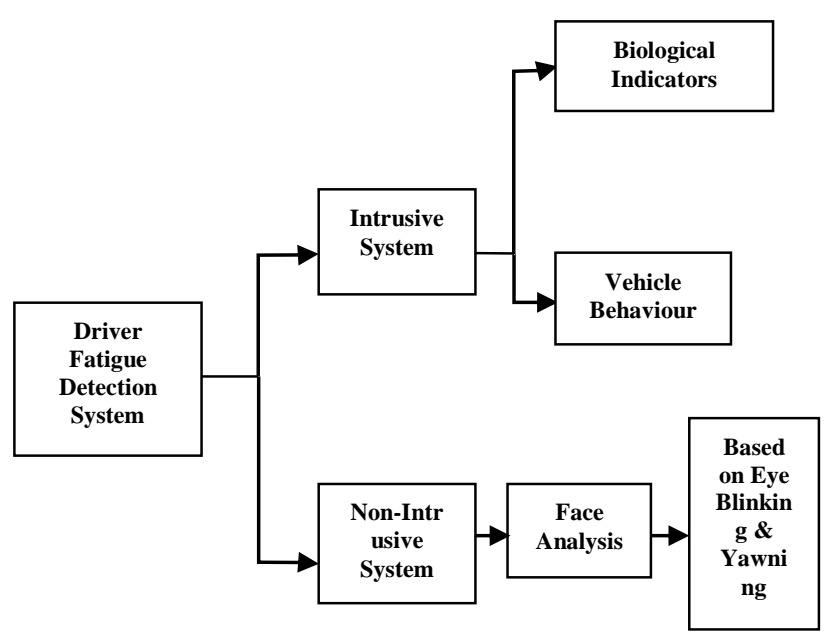

Fig.1. Types of Driver Fatigue Detection System

All the above discussed approaches are intrusive system of driver fatigue detection system. Few limitations of Intrusive methods are system is complex, cannot be placed easily, causes disturbance, poor performance, non-reliable, and produces noise. The solution is Non-Intrusive system.

The basic approach in non-intrusive system is analysis of face. The first symptom of fatigue appears in eye and than in mouth. Lot of research has been done to analyze the facial features to estimate driver fatigue based on eye blink rate and yawning.

Vijayalaxmi,et.al.,2012 proposed algorithm using Neural Network approach for eye detection is divided into two stages- training of neural network and detection. The Neural network is trained using GTAV and VITS database images. The success rate of the algorithm depends on training, more the number of images used for training more is the success rate.

The neural network is trained using two sets of imagesone set containing the object to be detected and other set 
consists of non-object images such as background, nose, eyebrows etc,. The success rate is $98 \%$ tested on GTAV database [23]. The algorithm is tested on images of different face orientation and works successfully on straight images but fails to detect both eyes if the face is oriented $90^{\circ}$. It detects at least one eye if the face is oriented by $30^{0}, 45^{\circ}$. The reason for failure of the algorithm on few images is improper ROI which leads to wrong identification of eyebrows as eyes.

Sudhakar.P.,et.al.,2011, proposed algorithm of face and eye detection both colour spaces are used. In this algorithm, face is extracted using skin information because in the input image only human face has skin region. The skin region is identified by converting RGB image to HSV (Hue, Saturation, Value). "Hue", gives basic pure colour of the image, "Saturation", gives how this pure colour is diluted by white light, "Value", gives information about intensity of an image. Using $\mathrm{H} \& \mathrm{~S}$ it is possible to get information about skin region. It is found that the range of $\mathrm{H}$ lies between 0.01 and 0.1 for skin. The success rate is $95.2 \%$ on Mathworks database images[24].

In 1998, researchers proposed non-intrusive driver fatigue system based on PERCLOS. Richard Grace, et al., used CCD camera, PC/104 processor, the method worked well for eye closure but problem with warning system [25].

In 2005, researchers proposed fatigue estimation using driver feedback, the method is non-intrusive but makes driver tiresome and annoying [2]. In 2006, Martin Gallagher used non-intrusive eye based $\&$ pressure sensor for fatigue detection. They used pressure sensor circuit along with $\mathrm{ADuC} 8031, \mathrm{PC}$, Camera. The success rate was $80 \%$, drawback was it takes 8 secs to process a frame and does not work on occluded face, different illumination condition[26].

Neeta Parmar [4], proposed driver drowsiness detection system based on eye blink rate, the success rate is $80 \%$.

The drawbacks of the system are poor illumination, unable to track eyes of person wearing spectacles, fails if one or both eyes are closed.

Martin Gallagher [5], proposed driver alert system for road safety, the problem with the system is it takes 8 secs to process each frame which is not desirable in real time implementation. Nidhi Sharma, et.al,[6], proposed driver drowsiness detection system using fuzzy logic. The drawback of the system is, it works only on still gray scale images and fails to track eyes of person wearing glasses.

In 2010, researchers made use of PERCLOS to detect driver fatigue, which detects eyes open, semi open, close. But time taken was more and required high grade PC to process the frames. In 2011, an author used Haar features to detect driver fatigue based on eye blink, the method works on rotated images with a success rate of $96 \%$ but fails to warn the driver and testing is done on computer.

The challenges for developing a driver fatigue detection systems are:

- System should be non-intrusive

- Selection of proper hardware

- Developing proper algorithm to detect and track face and eyes

- Perfect warning system

- Selecting proper programming language which can be easily interfaced to Embedded System

- Time taken to process the frame and alert the driver should be less

In this paper, a new approach is mentioned to estimate driver fatigue based on PERCLOS (Proportion/Percentage of time in a minute the eye is $80 \%$ closed). The rest of the paper is organized as Section 2 explains proposed algorithm for Fatigue estimation, Section 3 shows tested results under different illumination condition. Conclusions are drawn in Section 4.

\section{RELATED WORK- High PERFORMANCE AND LOW POWER DFD}

Driver fatigue is detected based on face analysis and calculating PERCLOS based on eyes. If the PERCLOS value exceeds $80 \%$ than driver is alerted to stop the vehicle. The proposed algorithm for fatigue detection using image processing is shown in Fig 2.

\section{A. Image Capture}

Logitech USB camera is used to capture the images at the rate of $30 \mathrm{fps}$ of size $480 \times 640$. The images are captured under different illumination condition for training Haar Cascade Classifiers and also for testing. The images are taken for different face orientation, distance and face occluded with glasses is shown in Fig 3.

\section{B. Haar Cascade Classifier}

To meet the challenges listed above, four different algorithms are tested in the laboratory for efficient detecting and tracking face and eyes. The methods developed are Template Matching, Skin Segmentation, Neural Networks and Haar Cascade Classifier. After

comparing all the four methods, it is observed that Haar Cascade Classifier achieves the highest success rate of $99.2 \%$.

Firstly Haar Cascade Classifier is trained for detecting face and eyes. To train two set of images are required i.e Positive images(images which contain object to be detected i.e face and eyes), Negative images (images which does not contain objects like scenery,pin,etc.).

In this research, total 4000 negative images and 3000 positive images from GTAV,Mathworks Video, Face expression and VITS database are used. As Viola and Jones suggested that Haar Cascade Classifier produces upto $95 \%$ detection rate if it is trained properly. In order to improve the efficiency of Haar Classifier, the positive images used for training are selected based on skin segmentation i.e, since positive images consists of objects to be detected, hence based on skin segmentation only face area is used for training by removing all other parts.

After training Haar Cascade Classifier, .xml file is generated which is loaded to detect and track face and eyes from captured images. Fig 4 shows, the results of success detection of Face and Eyes for different images. 


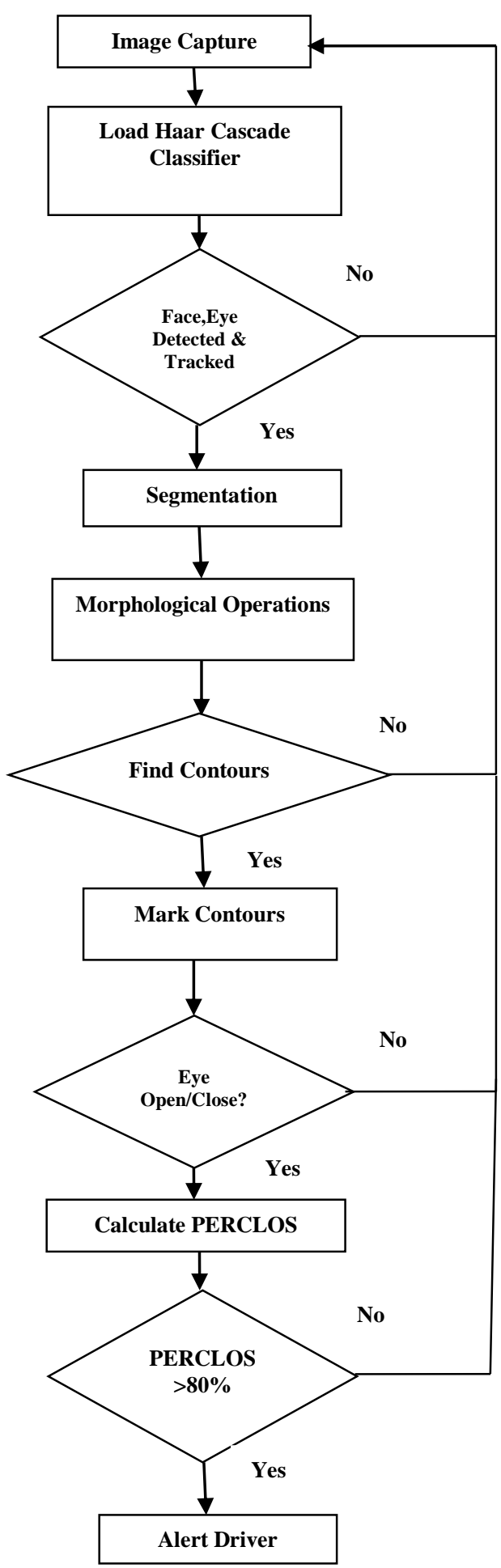

Fig.2. HPLP Driver Fatigue Detection System

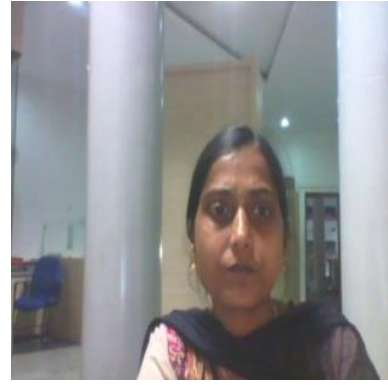

Straight image

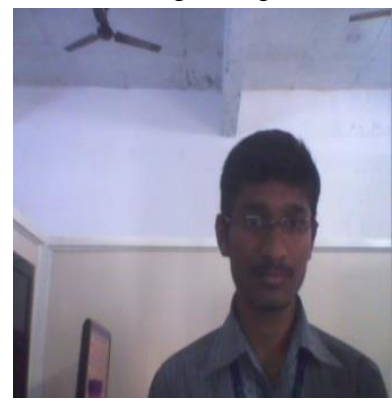

Image with Spectacles

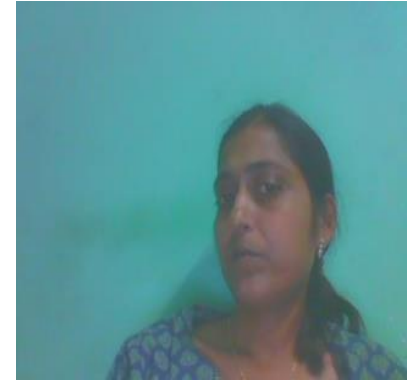

Head Rotated face image

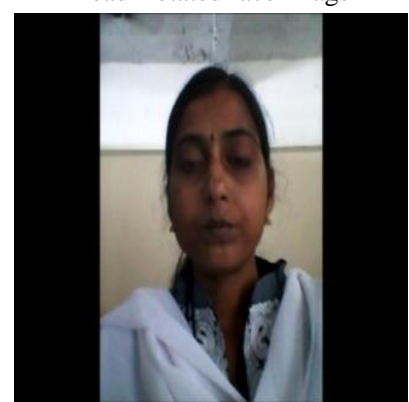

Closed eye image

Fig.3. Images captured under different Illumination

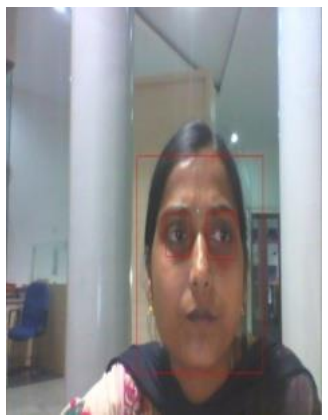

Face \& Eye detected

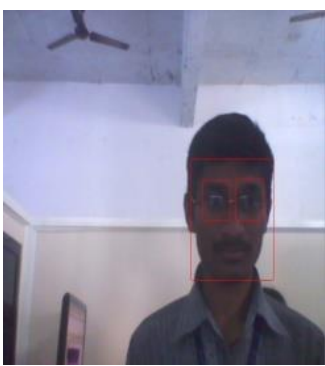

Face \& Eyes detected with spectacles

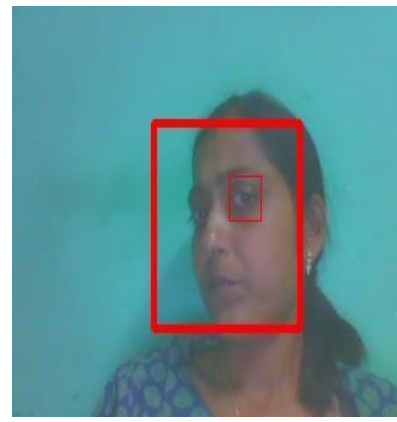

Face $\&$ one eye detected on rotate image

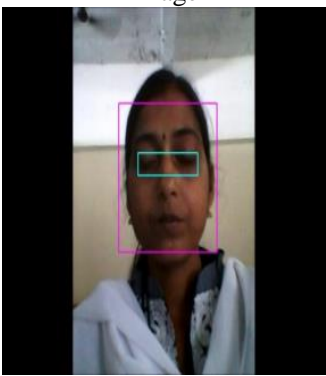

Face \& Eyes detected during closed eyes
Fig.4. Face \& Eyes detected \& tracked under different conditions

\section{Eye Open/Close}

After successful detection and tracking of Face and Eyes, next step is to find whether eyes are closed or open. The steps followed are

The original image size is $480 \times 640$, the size of the face is $222 \times 224$ and the sizes of cropped eyes are 147x64. 


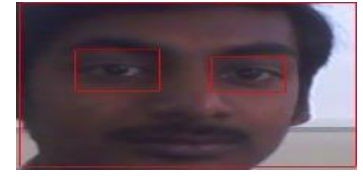

Face cropped image

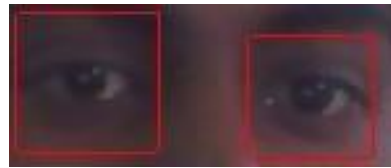

Eyes cropped
Fig.5. Eyes cropped

Image enhancement is the pre-processing step in image processing. There are so many methods for image enhancement, the approach used is Homomorphic filtering. The reason behind this is, most of driver fatigue detection system failed due to illumination effect. The image is a combination of illumination and reflectance, illumination is due to low frequency components and reflectance is due to high frequency component. Homomorphic filtering normalizes brightness across an image and increases contrast.

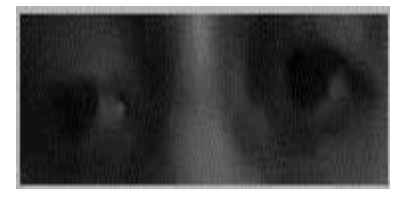

Grayscale image

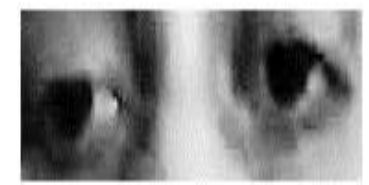

Enhanced eye image
Fig.6. Enhanced Eye image

It is a process of converting grayscale image to binary image. The binary image consists of only two gray levels, depending upon the threshold value

$$
\begin{gathered}
\mathrm{g}(\mathrm{x}, \mathrm{y})=0, \text { if } \mathrm{f}(\mathrm{x}, \mathrm{y})>\mathrm{T} \\
=1, \text { if } \mathrm{f}(\mathrm{x}, \mathrm{y})<\mathrm{T}
\end{gathered}
$$

where ' 0 ' represents Black and ' 1 ' represents White. In this the object i.e eye balls are assigned white pixel value and background is assigned with black pixel. Adaptive Thresholding is used in this algorithm, as it handles illumination problem more prominently compared to Global and Local Thresholding.

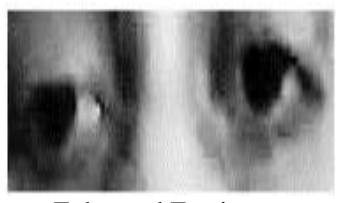

Enhanced Eye image

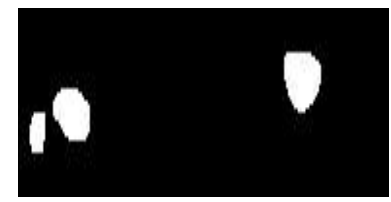

Binary Eye image
Fig.7. Binarized Eye image

Binary images contain numerous imperfections. Regions produced by Thresholding are distorted by noise and texture. Morphological operations remove these imperfections. It is a collection of non-linear operations related to the shape or morphology of features in an image. Dilation \& Erosion are the basic operations performed in morphology. Dilation allows objects to expand, thus potentially filling in small holes and connecting disjoint objects. Erosion shrinks objects by etching away (eroding) their boundaries. Erode operation is applied to remove noise from binary image.

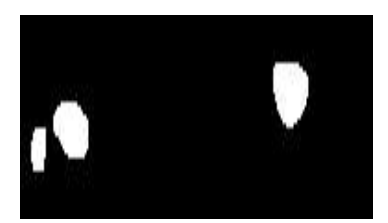

Binary image with noise

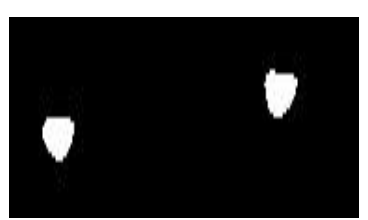

Binary image with noise removed
Fig.8. Binarized Eye image without noise

Contours are useful tool for shape analysis and object detection and recognition. For better results, the contours are applied on binary images where background should be black and white pixel represents object. Contour pixels are small subset of the total number of pixels representing a pattern. Amount of computation is greatly reduced. In this research Contours are used to find the white pixels and mark the contours.

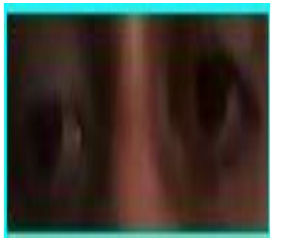

Open Eye image

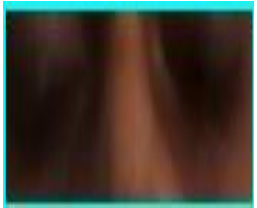

Closed Eye image

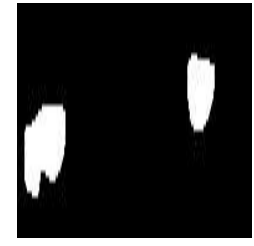

Binary image

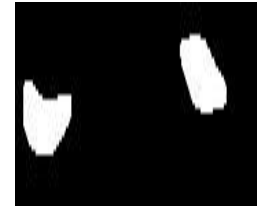

Binary image

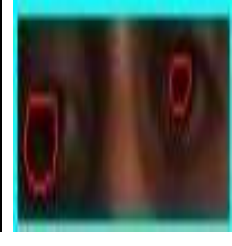

Contours

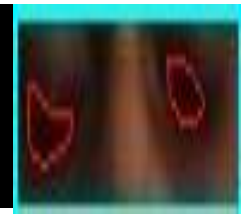

Contours
Fig.9. Open/Closed Eye images with contours

After marking contours, the number of white pixels representing contours is counted. If the value exceeds some fixed value then eyes are open otherwise eyes are closed. Since open eyes shows more white pixels compared to closed eyes.

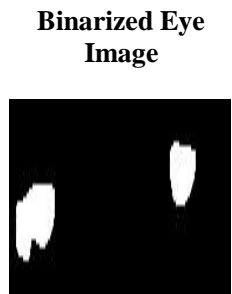

$\begin{array}{ccc}\text { Contours } & \begin{array}{c}\text { No.of White } \\ \text { Pixels }\end{array} & \begin{array}{c}\text { Status } \\ \text { of Eye }\end{array}\end{array}$
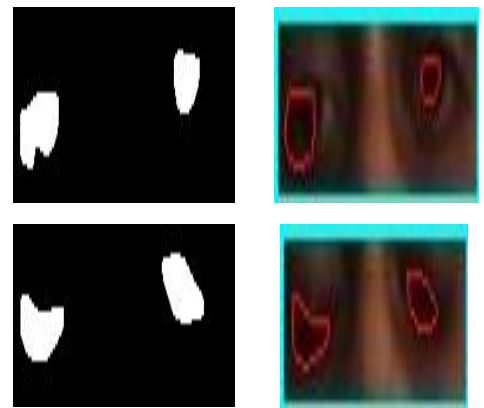

$\geq$ value

Open

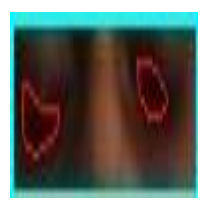

$<$ value

Close

Fig.10. Eye Open/Close images

PERCLOS is an established parameter to detect the level of drowsiness. The PERCLOS (the percentage of time that an eye is closed in a given period) score is measured to decide whether the driver is at drowsy state or not. On an average human blinks once every 5 seconds (12 
blinks per Minute).

Frame rate: $30 \mathrm{fps}$

In 60 Seconds: Total 1800 frames

Time Taken to read each frame: 0.0333 Secs

Number of frames with closed eyes: 15

Total time showing closed eyes: 0.4995 Secs

PERCLOS $=\left(0.4995^{*} 100\right) / 60$

$$
=0.8325 * 100=83.25 \%
$$

\section{RESULTS}

The complete set up is made in the laboratory and proposed algorithm is tested on four (GTAV, Face Expression, MathWorks Video and VITS) database images and live testing is also performed on 45 different persons under different illumination conditions. The setup is shown in figure below.

The results of the proposed algorithm are shown in figure, under different illumination conditions different head positions and for persons with and without glasses. The images used for testing the proposed algorithm are tabulated in Table 1.

Table 1. Images used for Testing the System

\begin{tabular}{|c|c|c|}
\hline S.N. & Database & Total No. of Images \\
\hline 1 & Face Expression & 148 \\
\hline 2 & GTAV & 46 \\
\hline 3 & MathWorks Video & 126 \\
\hline 4 & VITS & 200 \\
\hline 5 & Live & $\begin{array}{c}45(15 \text { Women, 15 Men, 15 } \\
\text { persons wearing glasses }\end{array}$ \\
\hline
\end{tabular}

The overall success rate of the proposed system is calculated using equation (1) given below

$$
\text { Overall Detection }(\%)=\frac{\mathrm{TP}+\mathrm{TN}}{\mathrm{TP}+\mathrm{TN}+\mathrm{FP}+\mathrm{FN}}
$$

Where

$\mathrm{TP}=$ True Positive: No. of frames that are correctly detected eye blinks.

FN= False Negative: No. of frames that show eye blinks but the program is not detected.

$\mathrm{FP}=$ False Positive: No. of frames that are reported as eye blinks but they are not.

TN=True Negative: No. of frames that are correctly reported as no blinks.

The system is tested on Video captured through Logitech USB camera and the details are shown in Table 2.
Table 2. Captured Video details for Testing

\begin{tabular}{|c|c|}
\hline Frame Rate & $30 \mathrm{fps}$ \\
\hline Frame Size & $480 \times 640$ \\
\hline Duration of the Video & $65 \mathrm{Sec}$ \\
\hline Total Frames & 1950 \\
\hline
\end{tabular}

$\mathrm{TP}=6, \mathrm{FP}=2, \mathrm{FN}=2, \mathrm{TN}=1890$

Overall Detection $=97.7 \%$

The proposed system is also Live Tested using Logitech USB camera and the details are shown in Table 3.

Table 3. Live Video details for Testing

\begin{tabular}{|c|c|}
\hline Frame Rate & $30 \mathrm{fps}$ \\
\hline Frame Size & $480 \times 640$ \\
\hline Duration of the Video & $85 \mathrm{Sec}$ \\
\hline Total Frames & 2550 \\
\hline
\end{tabular}

$\mathrm{TP}=27, \mathrm{FP}=4, \mathrm{FN}=2, \mathrm{TN}=2517$

Overall Detection $=\mathbf{9 9 . 7 \%}$

The simulation results of proposed Driver Fatigue Detection System tested in the laboratory are shown in Figure 11.
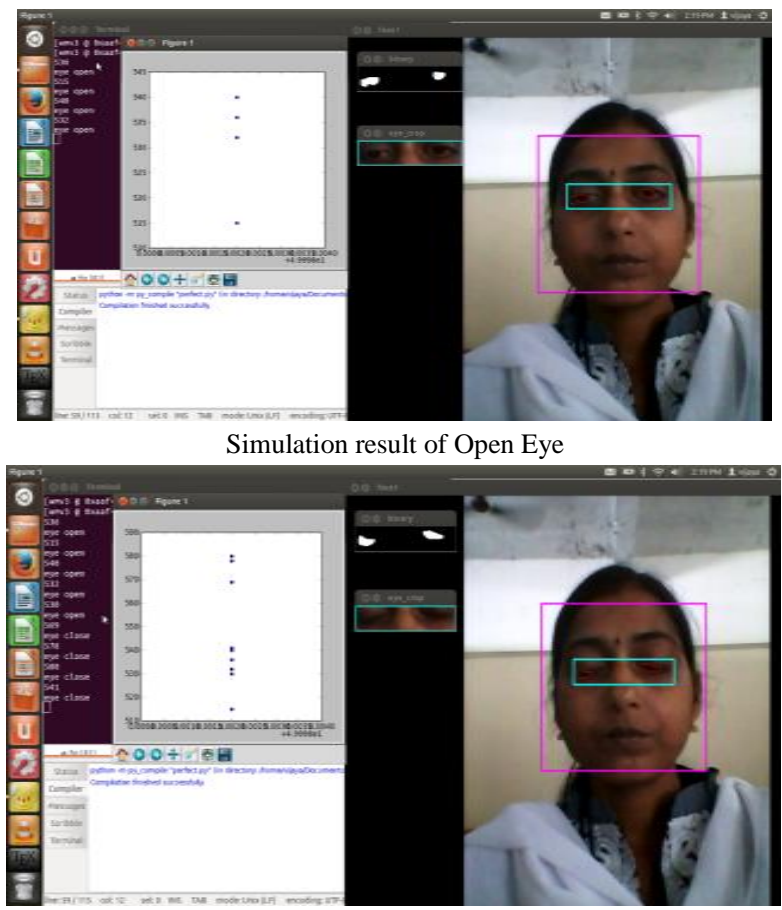

Simulation result of Closed Eye

Fig.11. Simulation Results 


\section{CONCLUSION}

In this paper a new system is proposed to estimate driver fatigue using image processing technique. Driver fatigue is estimated based on eye blink rate and is calculated using PERCLOS method. If the eyes are closed for 15 frames or if PERCLOS is $>80 \%$ than the driver is entering into drowsiness and driver is alerted to stop the vehicle. The face and eye are detected using Haar Cascade Classifier. Eye Open/Close is estimated using contours and counting the number of white pixels.

The entire set up is made in the laboratory and tested on GTAV, Mathworks Video, Face expression and VITS Database images. The system is tested on a video and the overall detection rate is $97.7 \%$. The system is also tested Live and the overall detection rate is $99.7 \%$. The developed algorithm works for different illumination, head rotation and persons wearing glasses. The time taken by the algorithm to estimate driver fatigue is $<5 \mathrm{~ms}$.

\section{REFERENCES}

[1] W. W. Wierwille, S. S. Wreggit, C. L. Kirn, L. A. Ellsworth, and R. J. Fairbanks III, "Research on vehicle-based driver status/performance monitoring: development, validation, and refinement of algorithms for detection of driver drowsiness," National Highway Traffic Safety Administration, U.S. DOT Tech Report No. DOT HS 808 247, 1994.

[2] Artaud et.al-1994, Mabbott et.al,-1999, Lavergne et.al, 1996, Vitabile et.al,2007-08, Eskandarian.A \& R.Sayed in 2005, "Monitoring the response of drivers.

[3] Boyraz.P.,Leicester,Hansen J.H.L, Sensing of Vehicle response, 2008.

[4] Neeta Parmar, Drowsy Driver Detection System, in 2002.

[5] Martin Gallagher, "Development of a driver alert system for road safety", in 2006-07.

[6] Nidhi Sharma, Prof. V. K. Banga, "Development of a Drowsiness Warning System based on the Fuzzy Logic", International Journal of Computer Applications (0975 8887) Volume 8- No.9, October 2010.

[7] M. J. Black, Y. Yacoob, Recognizing Facial Expressions in Image Sequences Using Local Parameterized Models of Image Motion. International Journal of Computer Vision. Vol. 25(1), October 1997, pp. 23 - 48; available online at http://citeseer.ist.psu.edu/black97recognizing.html.

[8] Jeffrey Huang, David Lie, Xuhui Shao, Harry Wechsler, Pose Discrimination and Eye Detection using SVM.

[9] M. Pardas, Extraction and Tracking of the Eyelids. International Conference on Acoustics, Speech and Signal Processing ICASSP 2000,4: 2357-2360, Istambul, Turkey, June $2000 \quad ; \quad$ available online at http://gpstsc.upc.es/imatge/pub/ps/ICASSP00_pardas.pdf

[10] S. Sirohey, A. Rosenfeld, Z. Duric, A method of detecting and tracking irises and eyelids in video, Pattern Recognition, Vol.35 (2002), pp.1389-1401; available online http://cs.gmu.edu/ zduric/WebPages/Papers/PR-2002-siro hey.pdf

[11] Zhiwei Zhu, Quing Ji, Robust real time eye detection and tracking under variable lighting conditions and various face orientations, Preprint submitted to Elseiver Science , July 5, 2004.

[12] Qiong Wang, Jingyu Yang, Eye detection in facial images with unconstrained background, Journal of pattern recognition research 1, 2006, published 25 sep 2006, pp.55-62.

[13] Qiong Wang, Jingyu Yang, Eye location \& eye state detection in facial images with unconstrained background, Journal of information and computing science, Vol.1, No.5, 2006,pp.284-289.

[14] Peng Wang, Matthew B, Green, Qiang Ji, James Wayman,Automatic Eye detection and its Validation,.

[15] Hyoung-Joon Kim and Whoi-Yul Kim, Eye detection in facial images using Zernike moments with SVM, ETRI journal, Vol.30, Nov,2,April 2008 pp. 335-337.

[16] Hawlader Abdullah Al-Mamun, Nadim Jahangir, Md. Shahedul Islam and Md. Ashraful Islam, Eye Detection in Facial Image by Genetic Algorithm Driven Deformable Template Matching. Vol.9, August,2009,

[17] Tanmay Raj pathak, Ratnesh kumar \& Eric Schwartz, Eye detection using morphological and colour image processing, Florida Conference on recent advances in Robotics, 2009, pp.1-6.

[18] Mihir Jain, Suman K.Mitra, Naresh D.Jotwani, Eye detection using line edge MAP template.

[19] Shylaja S S, K N Balasubramanya Murthy, S Natarajan, Nischith, Muthuraj R, Ajay S, Feed forward neural network based eye localization and recognition using hough transform, International journal of advanced computer science and applications, Vol.2, No.3, March-2011, pp.104-109.

[20] Rakhi C Motwani, Mukesh C Motwani,Dr.Frederick C. Harris,Jr., Eye Detection using Wavelets and ANN.

[21] Ms.Vijayalaxmi, Mr.Sreehari, "Knowledge based template for Eye detection", National Conference on Microwave,Antenna\&Signal Processing, pp.90 ,April 2011.

[22] Vijayalaxmi, P.Sudhakar, Sreehari, "Neural Network Approach for eye detection", The Second International Conference on Computer Science, Engineering and Applications (CCSEA-2012), May 26-27, Delhi, India, Proceedings Volume Editors: David C. Wyld, Jan Zizka, Dhinaharan Nagamalai ISBN : 978-1-921987-03-8,(2012).

[23] P.Sudhakar Rao, Vijayalaxmi, S.Sreehari, "New procedure for Segmenting Eyes from human Face", International Journal of Emerging Technologies and Applications in Engineering, Technology and Sciences, ISSN:0974-3588 July-Dec, Volume 4,Issue 2,(2011).

[24] Elsenbruch,S., Harnish,M., and Orr,W.C., "Heart rate variability during waking and sleep in healthy males and females," Sleep, Volume 22, pp.1067-1071, (1999).

[25] Martin Gallagher, "Development of a driver alert system for road safety", in (2006-07).

\section{Authors' Profiles}

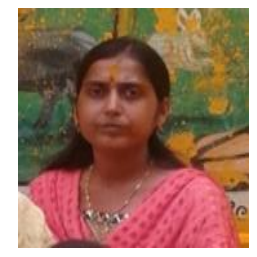

First Author Ms.Vijayalaxmi, received her B.E (E\&CE) degree from GNDEC, Visveswaraiah Technological University, Belgaum, Karnataka, India in 2003. M.Tech (DE\&CS) from JNT University, Hyderabad, Andhra Pradesh, India in 2008. She is pursuing her Doctorate from Gitam Institute of Technology, Gitam University, Vishakapatnam,Andhra Pradesh, India. Her area of research is Digital Image Processing. She has published 2 Journal papers, 3 International Conference papers and 2 National Conference papers. She received Best Paper award in August 2013 in IEEE International conference organized by Saveetha University, Chennai, India. She is the Life 
Member of ISTE and Member of IEEE.

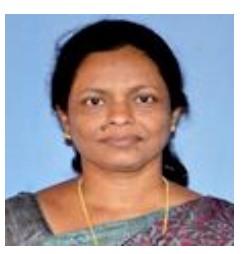

Second Author Dr. D.Elizabath Rani Completed Ph.D.,Electronics and Communication Engineering in 2003 from Anna University, India, Masters in Communication Systems in 1984 from Bharathiar University, India, Bachelors in Electronics and Communication Engineering in 1982 from Madurai Kamaraj, University, India. Presently she is Head of the Department of Electronics and Instrumentation branch in Gitam University, Vishakapatnam, India .She has 25 technical publications/ conference papers to her credit. She is a member of MISTE, IETE, SEMCE (I). She has 26 years of teaching experience and 15 years of research experience. Her area of interest are Signal processing, Communication systems, Network theory, Image processing.

How to cite this paper: Vijayalaxmi, D. Elizabeth Rani,"Driver Fatigue Estimation Using Image Processing Technique", International Journal of Information Technology and Computer Science(IJITCS), Vol.8, No.6, pp.66-72, 2016. DOI: 10.5815/ijitcs.2016.06.09 